\title{
A 12Bit 200Msps Spilt-Based Pipeline ADC Design
}

\author{
Haijun Lin, Hao San, and Ye Tian
}

\begin{abstract}
This paper presents a 12bit 200Msps pipeline ADC fabricated in TSMC 0.18um CMOS technology. For high resolution pipeline ADC design, the operation speed is limited by sampling capacitance load of OTA inside the ADC. The proposed ADC is realized in split-based pipeline architecture, sampling capacitance of ADC is separated into two channels. Each channel only has half capacitance, which reduce capacitive loading of OTAs in each channel and realize high speed operation of the ADC. The ADC achieves an SNDR of 64.7dB, SFDR of 86.3dB with analog input frequency of $10 \mathrm{MHz}$, sampling frequency of $100 \mathrm{MHz}$ and differential amplitude of $1.25 \mathrm{Vpp}$ without digital calibration. The power dissipation of ADC is $356 \mathrm{~mW}$ at $1.8 \mathrm{~V}$ supply.
\end{abstract}

Index Terms-OTA, pipeline ADC, split-based.

\section{INTRODUCTION}

CMOS ADCs with sampling rate more than 100Msps and resolution more than 10bit are widely used in optical communication system, wireless and wired broadband communication systems [1]. Pipeline is the appropriate architecture widely used in this class of ADCs for its reasonable trade-offs among sampling rate, resolution and power. To realize the high sampling rate and high resolution pipeline ADC, the OTA to drive large sampling capacitance inside MDAC becomes the bottleneck which limits the sampling rate of ADC. Split ADC architecture is the useful method to reduce the capacitance need to be driven by OTA in pipeline ADC [2]. Split-based pipeline ADC consists of 2 independent ADCs with the same circuit structure which shares a same SHA [3]. The block diagram of traditional split ADC is shown in Fig. 1, The output code of ADC (A) and $\mathrm{ADC}(\mathrm{B})$ are $X_{A}$ and $X_{B}$ respectively. By average calculation, the final output code of the ADC can be written as Eq. 1, and difference between outputs of $2 \mathrm{ADCs}$ can be written as Eq. 2.

$$
\begin{gathered}
X=\frac{X_{A}+X_{B}}{2} \\
\Delta X=X_{B}-X_{A}
\end{gathered}
$$

Manuscript received August 20, 2016; revised November 15, 2016. This work was supported in part by Science Foundation of Fujian Province under Grant 2014J01255 and Science and Technology Project of Fujian Provincial Department of Education under Grant JK2016027.

Haijun Lin is with the Fujian Provincial Key Laboratory of Opto-electronic Technology and Devices, Xiamen University of Technology, Xiamen, Fujian, China (e-mail: linhaijun@xmut.edu.cn).

Hao San is with the Department of Information and Communication Engineering, Tokyo City University, Tokyo, Japan (e-mail: hsan@tcu.ac.jp).

Ye Tian is with the Xiamen Xunxin Electronic Technology Co., LTD., Xiamen, China (e-mail: 11130577@qq.com).
In Eq. 2, $\triangle X$ is the output of two channel ADC difference due to mismatch between two ADCs. This value can be used to calibrate the channel mismatch such as capacitance mismatch and OTA gain mismatch between ADC (A) and ADC (B).

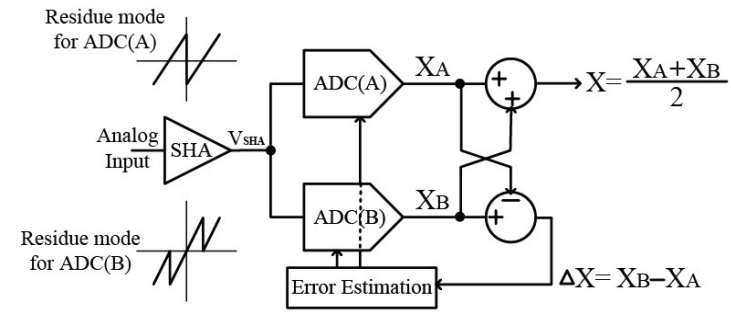

Fig. 1. The traditional split ADC block diagram.

Several digital calibration algorithms for split ADC are proposed [1]-[6]. But for high speed pipeline ADC design, the digital calibration for split ADC has three difficulties:

1) In Fig. 1, the residue transfer curve is different between two ADCs. Which means the different reference voltage is required for two ADCs for calibration.

2) In Fig. 2, random sequences should be injected to ADC. $q_{1 \mathrm{~A}}, q_{1 \mathrm{~B}}, q_{2 \mathrm{~A}}, q_{2 \mathrm{~B}}$ are examples of random sequence injected to ADC. Calibration time is very long, which limits ADC production throughput, the reliability of the random sequences injection is not proved by industry [5].

3) In Fig. 2, while random sequences are injected to ADCs for calibration, extra switches should be added into signal paths, the additional switch $\mathrm{ON}$ resistance increases $\mathrm{RC}$ time constant in signal path, which will decrease the operation speed of ADC.

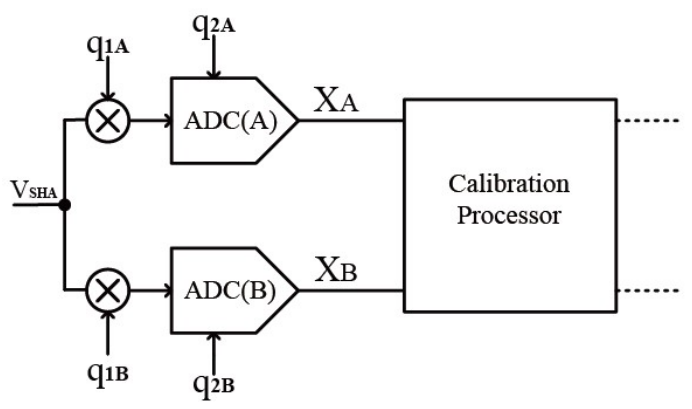

Fig. 2. Example of split $\mathrm{ADC}$ with random sequence.

Split ADC is not be used for high speed ADC for difficulties discussed above. To apply this architecture on high speed ADC, split-based pipeline ADC without digital calibration is proposed, no extra switches in signal paths, OTAs in each ADC only drive half capacitance, which relax design requirement of OTAs and increase the operation speed of ADC. Since only one SHA used for two channel ADCs, 
clock mismatch between channels need not be considered. The proposed split-based pipeline ADC operates with sampling frequency of $200 \mathrm{MHz}$ and resolution of $12 \mathrm{bit}$ can be confirmed.

The architecture of proposed split-based pipeline ADC is discussed in Section II. The circuit design is introduced in Section III. Measurement results are discussed in Section IV and Section V summarizes and concludes this paper.

\section{ARChiteCtURE of Proposed ADC}

Traditional 12bit pipeline ADC is shown in Fig. 3 [7], which is composed of five 2.5 bit stages and a 2 bit flash converter. In Fig. 3, $V_{\text {inp }}$ and $V_{\text {inn }}$ are analog input differential signals of ADC. To realize resolution of 12 bit and sampling frequency of $200 \mathrm{MHz}$, from calculation and simulation, the design requirement of OTAs in each 2.5bit sub-ADC stages is shown in Table I.

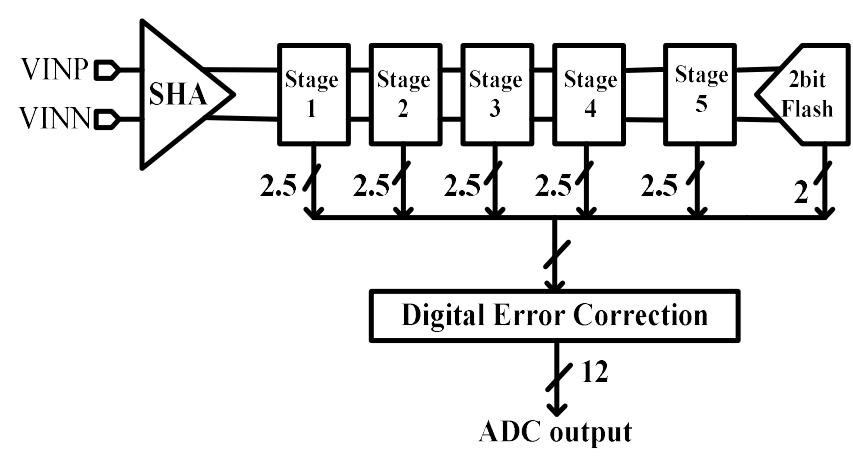

Fig. 3. Traditional 12bit Pipeline ADC architecture.

\begin{tabular}{lccccc}
\multicolumn{5}{c}{ TABLE I: DESIGN REQUIREMENT OF OTA IN EACH SUB-ADC STAGE } \\
\hline \hline Parameters & OTA1 & OTA2 & OTA3 & OTA4 & OTA5 \\
\hline DC gain (dB) & 78 & 68 & 56 & 44 & 32 \\
GBW $(\mathrm{GHz})$ & 3.7 & 3.2 & 2.6 & 2.1 & 1.6 \\
Load $(\mathrm{pF})$ & 2 & 0.75 & 0.38 & 0.18 & 0.05 \\
\hline \hline
\end{tabular}

From Table I, it is clear that the design requirements on OTAs in each stage are very rigor. The operation speed of OTA is depends on sampling capacitance be driven by OTA. Large output current from OTA is required to drive large sampling capacitance for high speed operation, which increases power dissipation. In order to realize high gain of OTA, large $\mathrm{g}_{\mathrm{m}}$, hence large $\mathrm{W} / \mathrm{L}$ is required. However, the large gate parasitic capacitor of MOSFET at input of OTA will limit the sampling speed of ADC. To realize high speed sampling, split-based pipeline ADC is proposed.

Fig. 4 shows the basic idea of split-based ADC, Fig. 4 (a) shows an $N$ bit ADC with single channel, $g_{m}$ means the transconductance of OTA and $\mathrm{C}$ means the total capacitance, the last block means the digital circuit in ADC. Because the bandwidth $f_{T}$ of the OTA is proportional to $g_{m} / \mathrm{C}$, power $\mathrm{P}$ is proportional to $\mathrm{g}_{\mathrm{m}}$. In the other hand, in a split based ADC (Fig. 4 (b)), OTAs in each ADC channel has half $g_{m}$, half load and the half power with the same bandwidth, thermal noise will increase $3 \mathrm{~dB}$ in each channel since capacitance is half compared with the single ADC. However, since output of two channel ADCs be averaged, increased noise is recovered in the output. The digital output $D_{\text {out }}$ can be written as
$D_{\text {out }}=\left(D_{\text {out }}(\mathrm{A})+D_{\text {out }}(\mathrm{B})\right) / 2$. As the result, the bandwidth, noise level, and total power of split ADC is the same as the single ADC.

The proposed spilt-based pipeline ADC is shown in Fig. 5. The ADC (A) and ADC(B) are the same ADC with five 2.5bit sub-ADCs and a 3bit flash ADC for considering linearity, maximum conversion rate, power consumption and chip area. Redundancy correction of sub-ADC output code and average calculation of two channel ADCs are processed with on-chip digital block. Digital calibration is not used in proposed spilt-based pipeline ADC for problems of calibration time and operation speed.

The split-based pipeline ADC relaxes the design requirement on each $\mathrm{ADC}$ especially for OTA, so that this architecture is suitable for high speed high resolution pipeline ADC implementation. Compared to time interleaved ADC, split-based ADC uses a single SHA to eliminate the effect of sampling phase inaccuracy among each ADC channels. Also this proposed architecture can be expanded to four channels and more than four channels application.
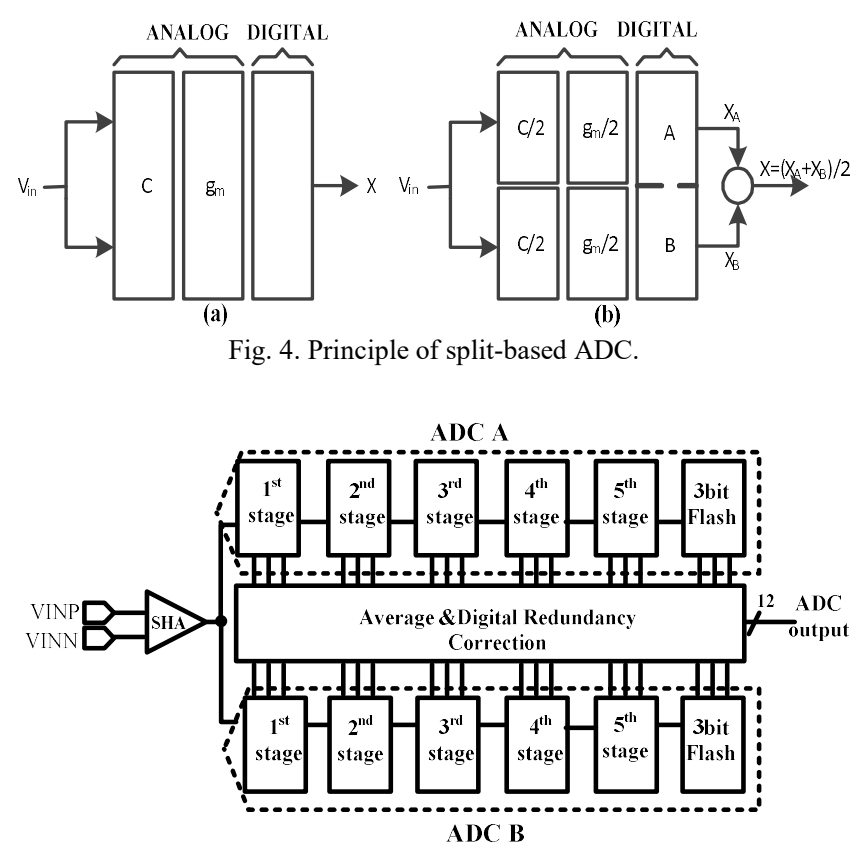

Fig. 5. Proposed split-based ADC architecture.

\section{CIRCUIT IMPLEMENTATION}

The proposed split-base 12 bit $200 \mathrm{Msps}$ pipeline ADC is composed of SHA, split-based ADC, band-gap circuit, reference generator, DLL, clock distribution circuit, LVDS and digital control circuit. The block diagram of proposed ADC is shown in Fig. 6.

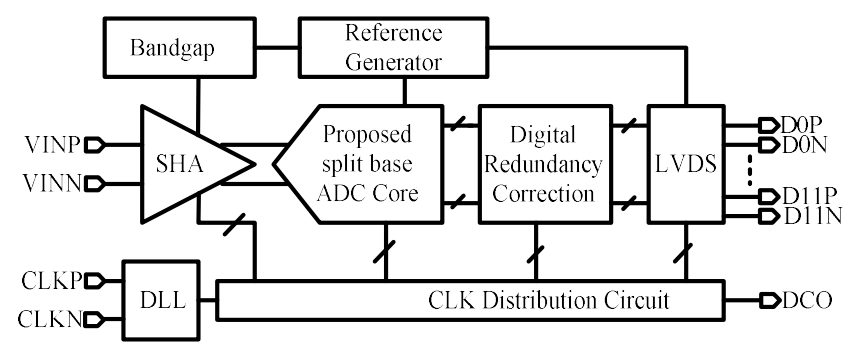

Fig. 6. The Block Diagram of Proposed ADC. 
SHA is used to sample and hold input analog signal, band-gap circuit generates reference signal and common mode signal of system, reference generator makes reference signal of comparator of ADC, DLL generates stable clock signals to ADC, Clock distribution circuit builds clock signals required by SHA, proposed $\mathrm{ADC}$ core, digital redundancy correction, LVDS. LVDS drives 12 bit digital output data (from $D_{0 \mathrm{p}}$ to $D_{11 \mathrm{~N}}$ ). DCO is the output clock used for output data capture.

In this section, the design of SHA and 2.5 bit ADC stage are introduced.

\section{A. SHA}

Sampling and hold amplifier (SHA) is the crucial part in a high speed and high resolution ADC, ADC performance such as dynamic range, distortion, SFDR and noise are largely dependent on SHA. In our implementation, wide band and low noise, high linearity SHA is required. The conventional two-capacitor flip around SHA architecture is appropriate selection. Fig. 7 shows the block diagram of the SHA. To reduce the charge injection and clock feed-through effect, bottom plate sampling technique is used. Sampling capacitor $C_{s}$ is $4 \mathrm{pF}$ considering the required $12 \mathrm{bit}$ accuracy and $\mathrm{kT} / \mathrm{C}$ noise at $1.25 \mathrm{~V}_{\mathrm{p}-\mathrm{p}}$ full scale input, total capacitors for differential SHA is $4 \mathrm{pF}$. The total capacitive load of SHA is sampling capacitors in 1st stage of two channel ADCs.

To reduce the $\mathrm{ON}$ resistance and nonlinearity of switches, $S_{1}, S_{2}, S_{1 \mathrm{a}}$ are chosen as bootstrapped switches.

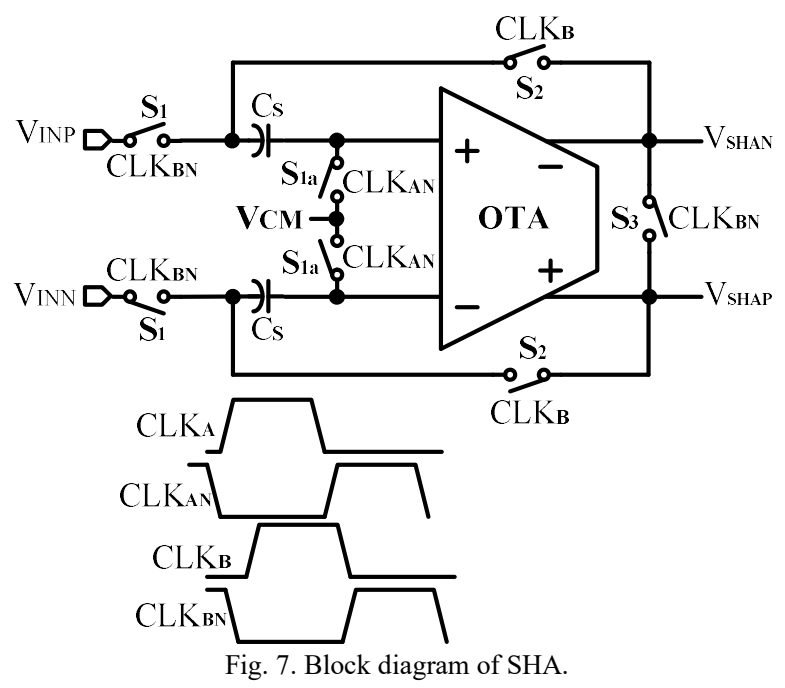

Clocks timing of SHA shows in Fig. 7, the rise edge of $\mathrm{CLK}_{\mathrm{BN}}$ is the sampling phase and the fall edge of CLKB is the holding phase of SHA respectively.

Achieving sufficient dc gain at a high sampling rate with low power is a difficult challenge for OTA design. Although a multistage OTA offers high open-loop gain, the requirement of frequency compensation increases the OTA power. Single stage OTA offers large gain-bandwidth products with limited $\mathrm{dc}$ gain. To realize high gain, wide bandwidth and high drive capability, two-stage amplifier with gain boost foldedcascode architecture is employed. Fig. 8 shows the OTA circuit, the input stage is designed by PMOS, which induces thermal noise. Switched-capacitor common mode feedback is used to stabilize the common mode voltage.

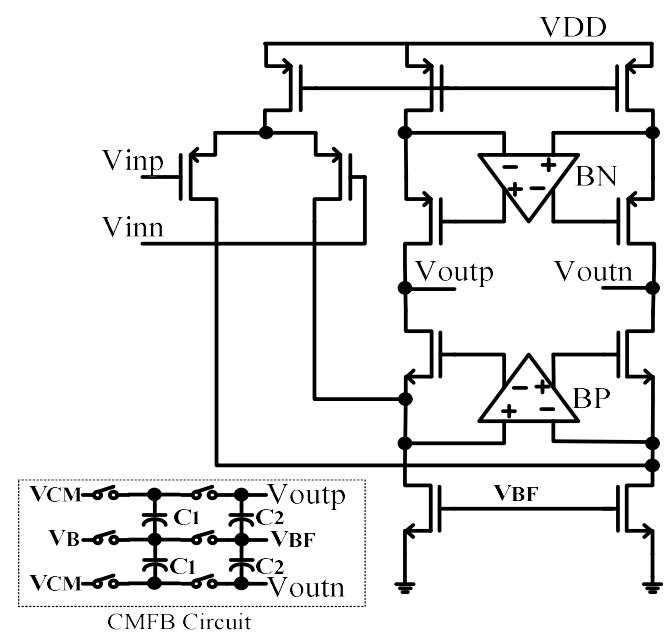

Fig. 8. OTA circuit used in SHA.

Simulated performance results of the OTA show in Table II.

TABLE II: SIMULATION RESULTS OF OTA IN SHA

\begin{tabular}{llllll}
\hline \hline Parameters & Input swing & CM Voltage & DC gain & GBW & SFDR \\
\hline Value & $1.25 \mathrm{~V}_{\mathrm{p}-\mathrm{p}}$ & $0.9 \mathrm{~V}$ & $79 \mathrm{~dB}$ & $1 \mathrm{GHz}$ & $88 \mathrm{~dB}$
\end{tabular}

\section{B. Pipeline Stage with 2.5bit MDAC}

The proposed split-based pipeline ADC is implemented by 2.5bit MDAC, which includes six comparators, four switched capacitor circuit paths to realize charge calculation. The single-ended diagram of the $1^{\text {st }}$ stage 2.5 bit MDAC shows in Fig. 9.

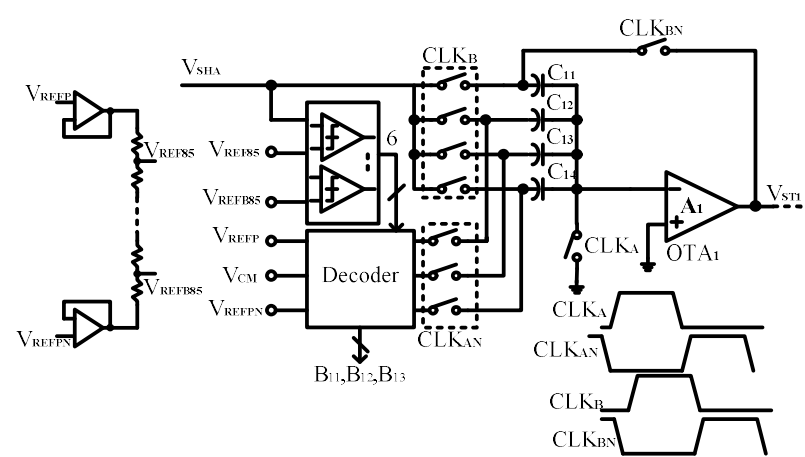

Fig. 9. Single-ended diagram of the $1^{\text {st }}$ stage 2.5bit MDAC.

$V_{\mathrm{SHA}}$ is the input signal of $1^{\text {st }}$ stage MDAC, which is the output of SHA, $V_{\text {REFP }}$ and $V_{\text {REFN }}$ are positive and negative reference voltage respectively and are created by reference generator, and reference voltage such as $V_{\text {REF85 }}$ and $V_{\text {REFB85 }}$ are reference voltage for comparators, which are created by resistor ladder inside the MDAC. When $\mathrm{CLK}_{\mathrm{B}}$ is high, MDAC is at sampling phase, $V_{\mathrm{SHA}}$ is charged to $C_{11}$ to $C_{14}$; in the non-overlapping phase between $\mathrm{CLK}_{\mathrm{B}}$ and $\mathrm{CLK}_{\mathrm{BN}}$, digital outputs from comparators are transferred to the decoder. According to the decoder outputs, one of the voltage references $\left(\mathrm{V}_{\mathrm{REFP}}, \mathrm{V}_{\mathrm{CM}}\right.$ and $\left.\mathrm{V}_{\mathrm{REFN}}\right)$ is chosen. When $C L K_{\mathrm{AN}}$ turns high, these values are fed to the bottom plate of $C_{12}, C_{13}$ and $C_{14}$ respectively. When $\mathrm{CLK}_{\mathrm{BN}}$ is high, MDAC is at subtraction and hold phase, charge on $C_{12}, C_{13}, C_{14}$ are transferred to $C_{11}$, produces the residual output voltage to next stage. 
The Default value of capacitors for 1 st stage Sub-ADC is $C_{11}=C_{12}=C_{13}=C_{14}=0.5 \mathrm{pF}$. Total capacitive loads for SHA are $4 \mathrm{pF}$ includes $\mathrm{ADC}(\mathrm{A})$ and $\mathrm{ADC}(\mathrm{B})$.

The comparator of the 2.5 bit MDAC is the 4 inputs full differential comparator. Which used to compare differential input and reference signal, output the compared value. Outputs of six comparators are formed as binary codes, become digital outputs $\mathrm{B}_{11}, \mathrm{~B}_{12}, \mathrm{~B}_{13}$. Comparator circuit is shown in Fig. 10.

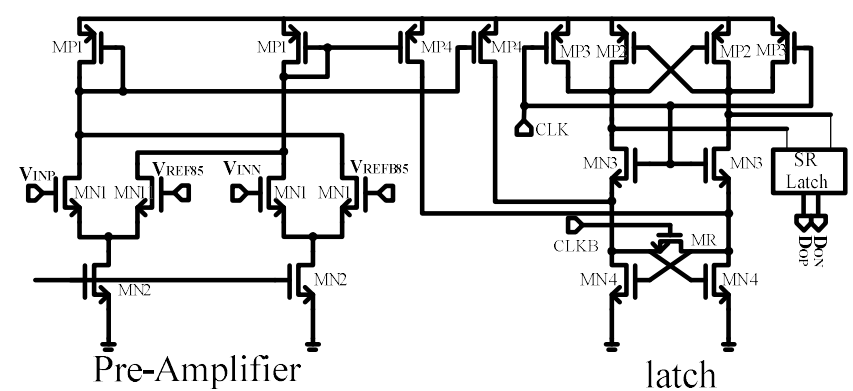

Fig. 10. Comparator circuit.

The comparator composed of pre-amplifier, latch and SR latch circuit. Pre-amplifier is to amplify small difference signal between $\left(\mathrm{V}_{\text {INP }}-\mathrm{V}_{\text {INN }}\right)$ and $\left(\mathrm{V}_{\mathrm{REF} 85}-\mathrm{V}_{\mathrm{REFB85}}\right)$. Here $\mathrm{V}_{\text {INP }}$ and $V_{\text {INN }}$ are positive and negative held signals from SHA. $\mathrm{V}_{\text {REF85 }}$ and $\mathrm{V}_{\text {REFB85 }}$ is one pair of positive and negative reference signals built by reference buffer. This amplified difference signal is latched when CLK turns high, latch operates as a positive feedback. In this phase, latch generates the output and feeds it to SR latch. When CLKB turns high, comparator works in reset phase. SR latch works as a driver to generate CMOS levels and drives the digital logic gates in the decoder block.

\section{EXPERIMENTAL RESULTS}

The proposed split-based pipeline ADC is designed and fabricated in TSMC 0.18um CMOS technology and a nominal supply of $1.8 \mathrm{~V}$. The die micrograph of the proposed $\mathrm{ADC}$ is shown in Fig. 11. The active area is $3 \times 4 \mathrm{~m}^{2}$.

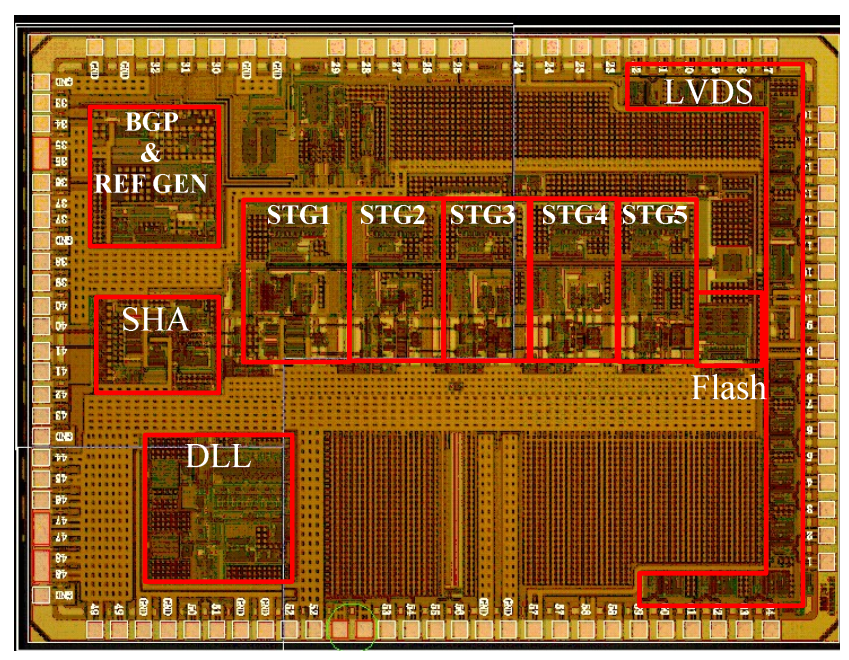

Fig. 11. Micrograph of Proposed ADC.

During chip measurement, the input signal and clock signal are obtained by R\&S SMA 100A and Si530 chip respectively.
Both were filtered using high order passive band-pass filters around the applied frequency to remove harmonics and white noise produced by signal sources.

Fig. 12 shows the measured static performance of proposed ADC. The ADC differential non-linearity (DNL) and integral non-linearity (INL) are $-0.67 /+0.62 \mathrm{LSB}$ and $-0.79 /+0.85$ LSB respectively. This performance is obtained without digital calibration technique.
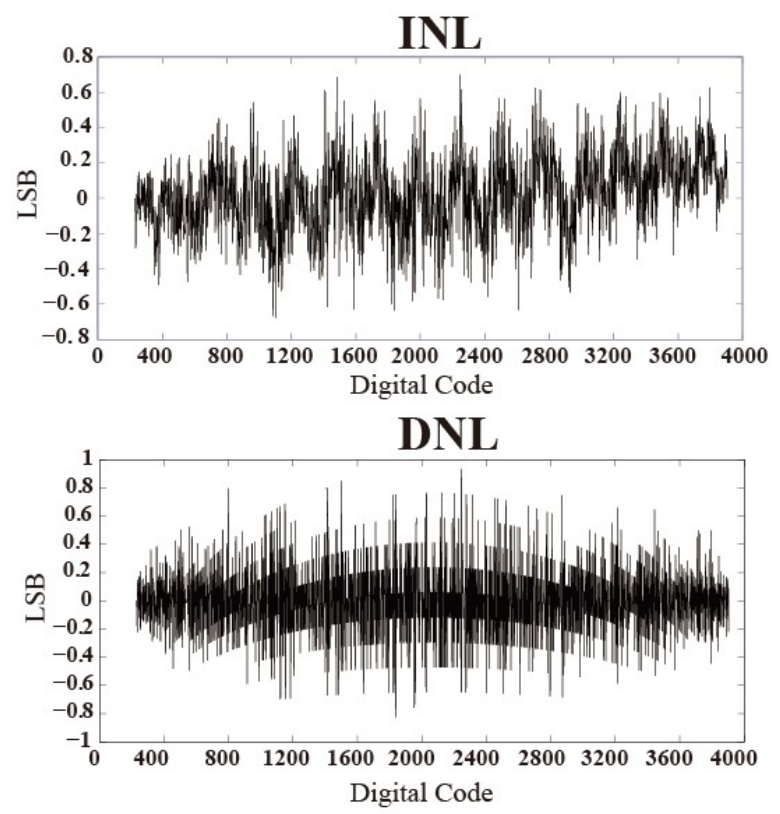

Fig. 12. Measured INL and DNL of proposed ADC.

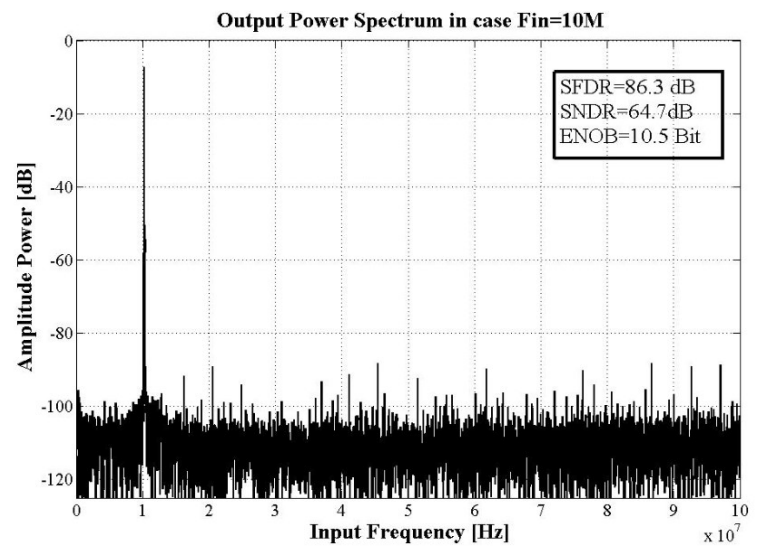

(a)

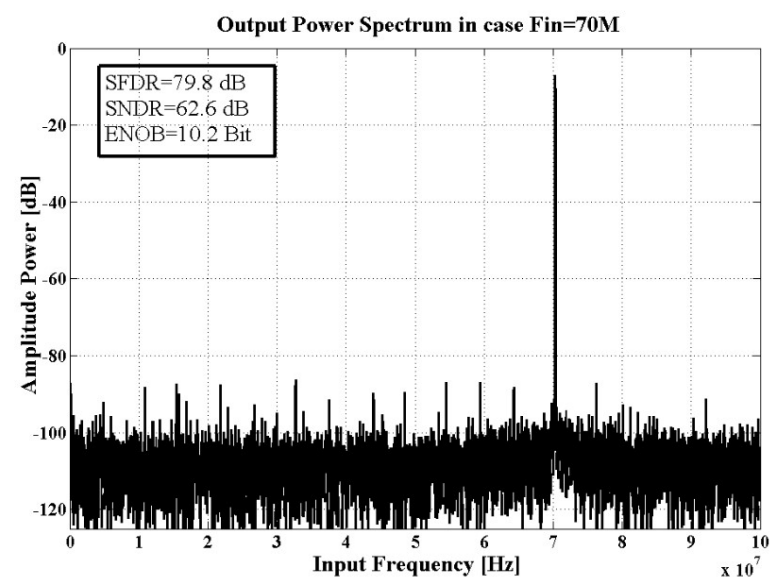

(b)

Fig. 13. Output spectrum in case (a) $F_{\text {in }}=10 \mathrm{MHz}$, (b) $F_{\text {in }}=70 \mathrm{MHz}$ with sampling frequency of $200 \mathrm{MHz}$. 
Fig. 13 shows the measured output power spectrum of $\mathrm{ADC}$ at sampling frequency of $200 \mathrm{MHz}$ and input signal amplitude power of -1dBFS. Fig. 13 (a) gives the dynamic performance of ADC with input signal frequency of $10 \mathrm{MHz}$. Parameters such as SFDR is $86.3 \mathrm{~dB}$, SNDR is $64.7 \mathrm{~dB}$ and ENOB is 10.5 bit are measured. Fig. 13 (b) shows the performance with input signal frequency of $70 \mathrm{MHz}$. Parameters such as SFDR is $79.8 \mathrm{~dB}$, SNDR is $62.6 \mathrm{~dB}$ and ENOB is 10.2 bit are measured. As indicated in Fig. 14, the proposed $\mathrm{ADC}$ dynamic performance declines from input signal frequency from $10 \mathrm{MHz}$ to $70 \mathrm{MHz}$. the SFDR drops from $86.3 \mathrm{~dB}$ to $79.8 \mathrm{~dB}$. The reason for this decline is the length of bonding wire of the package is more than 2 millimeters, the parasitic inductance of bonding wire decreases the quality of input signal. This parasitic effect becomes serious along with input signal increases. Also the parasitic inductance from bonding wire of power supply induces high frequency noise, which decreases noise dynamic performance of ADC too. This ADC consumes $356 \mathrm{~mW}$ with power supply of $1.8 \mathrm{~V}$. The power distribution of ADC shows in Table III, Table IV summarizes the measured performance of the proposed split-based pipelined ADC and Table V summarizes the performance of this work compares with published papers of pipelined ADCs.

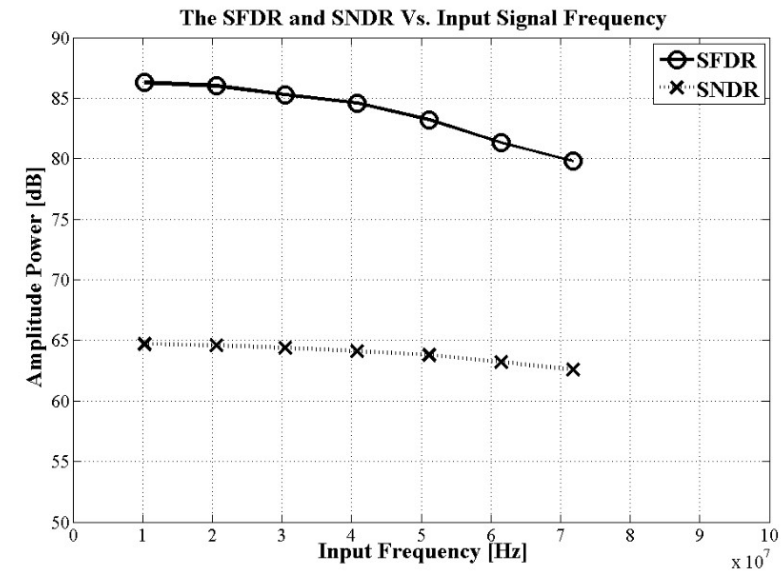

Fig. 14. Measured dynamic performance versus input frequency at 200Msps sampling rate.

TABLE III: THE POWER DISTRIBUTION OF ADC

\begin{tabular}{ll}
\hline \hline Circuit component & Power $(\mathrm{mW})$ \\
\hline SHA & 54 \\
Split-based ADC core & 120 \\
Band-gap \& reference & 90 \\
generator & 20 \\
DLL & 50 \\
Clock Distribution circuit & 22 \\
Digital control circuit & 356 \\
Power dissipation &
\end{tabular}

TABLE IV: MEASUREMENT PERFORMANCE OF PROPOSED ADC

\begin{tabular}{ll}
\hline Parameters & Value \\
\hline Process & $0.18 \mathrm{um} 1 \mathrm{P} 6 \mathrm{M} \mathrm{CMOS}$ \\
Power supply & $1.8 \mathrm{~V}$ \\
Sampling Frequency & $200 \mathrm{MHz}$ \\
Input Frequency & $10 \mathrm{MHz}-100 \mathrm{MHz}$ \\
Input signal range & $1.25 \mathrm{~V}_{\mathrm{p}-\mathrm{p}}$ \\
SNDR@10MHz & $64.7 \mathrm{~dB}$ \\
$\quad 670 \mathrm{MHz}$ & $62.6 \mathrm{~dB}$
\end{tabular}

\begin{tabular}{|c|c|}
\hline SFDR@10MHz & $86.3 \mathrm{~dB}$ \\
\hline @70MHz & $79.8 \mathrm{~dB}$ \\
\hline INL@10MHz & $-0.67 /+0.62 \mathrm{LSB}$ \\
\hline DNL@10MHz & $-0.79 /+0.85 \mathrm{LSB}$ \\
\hline Clock jitter & $0.5 \mathrm{ps}_{\mathrm{rms}}$ \\
\hline Power dissipation & $356 \mathrm{~mW}$ \\
\hline
\end{tabular}

TABLE V: PERFORMANCE COMPARISON

\begin{tabular}{llll}
\hline \hline & This work & {$[8]$} & $9]$ \\
\hline Process & $0.18 \mathrm{um}$ CMOS & $90 \mathrm{~nm}$ CMOS & $55 \mathrm{~nm}$ CMOS \\
Power supply & $1.8 \mathrm{~V}$ & $1.2 \mathrm{~V}$ & $1.1 \mathrm{~V}$ \\
Resolution & $12 \mathrm{bit}$ & $12 \mathrm{bit}$ & $12 \mathrm{bit}$ \\
Sampling & $200 \mathrm{Msps}$ & $200 \mathrm{Msps}$ & $200 \mathrm{Msps}$ \\
rate & & $64 \mathrm{~dB}$ & $64.6 \mathrm{~dB}$ \\
SNDR & $64.7 \mathrm{~dB}$ & & $82.9 \mathrm{~dB}$ \\
SFDR & $86.3 \mathrm{~dB}$ & $-1.70 /+1.30$ & $-1.89 /+1.36$ \\
INL(LSB) & $-0.67 /+0.62$ & $-0.59 /+0.87$ & $-0.28 /+0.24$ \\
$\begin{array}{l}\text { DNL (LSB) } \\
\text { Power }\end{array}$ & $-0.79 /+0.85$ & $348 \mathrm{~mW}$ & $30.7 \mathrm{~mW}$ \\
$\begin{array}{l}\text { dissipation } \\
\text { Digital }\end{array}$ & $356 \mathrm{~mW}$ & With & With \\
calibration & Without & & \\
\hline \hline
\end{tabular}

As performance comparison shown in Table $\mathrm{V}$, the proposed split-based ADC has the best SNDR, SFDR performance with low power dissipation without digital calibration.

\section{CONCLUSION}

This paper describes a split-based 12bit 200Msps pipeline ADC without digital calibration. The proposed ADC distributes sampling capacitances of pipeline ADC into 2 $\mathrm{ADC}$ channels, which relaxes requirement of OTA inside each ADC and improves conversion rate of each ADC. The proposed split-based ADC architecture realized high resolution $\mathrm{ADC}$ with high conversion rate. Although mismatch between 2 channels reduces the SFDR of ADC, 12 bit resolution can be obtained by careful layout. The chip measurement results confirmed the proposed split-base pipeline ADC achieves SFDR of $86.3 \mathrm{~dB}$, SNDR of $64.7 \mathrm{~dB}$ with input signal frequency of $10 \mathrm{MHz}$ and sampling frequency of $200 \mathrm{MHz}$. The power dissipation is $356 \mathrm{~mW}$. The proposed split-base pipeline ADC can also be applied to design 4 channels $\mathrm{ADC}$ to improve sampling rate speed. It is our future work.

\section{ACKNOWLEDGMENT}

Thanks Professor Haruo Kobayashi of Gunma university of Japan for his valuable advice.

\section{REFERENCES}

[1] J. Arias, V. Boccuzzi, and L. Quintanilla, "Low power pipeline ADC for Wireless LANs," IEEE Journal of Solid State Circuits, vol. 39, no. 8, pp. 1338-1340, 2004.

[2] J. A. McNeill, M. C. W. Coln et al., ""Split ADC" architecture for deterministic digital background calibration of a 16-bit 1-MS/s ADC," IEEE Journal of Solid-State Circuits, vol. 40, no. 12, pp. 2437-2445, 2005.

[3] I. Ahmed and D. A. Johns, "An 11-Bit $45 \mathrm{MS} / \mathrm{s}$ pipelined ADC with rapid calibration of DAC errors in a multi-bit pipeline stage," IEEE Journal of Solid-State Circuits, vol. 43, no. 7, pp. 1626-1637, 2008. 
[4] J. L. Fan, C. Y. Wang, and J. T. Wu, "A robust and fast digital background calibration technique for pipelined ADCs," IEEE Transactions on Circuits and Systems, vol. 54, no. 6, pp. 1213-1223, 2007.

[5] J. A. McNeill et al., "Digital background-calibration algorithm for "Split ADC" architecture," IEEE Journal of Solid-State Circuits, vol. 56, no. 2, pp. 294-306, 2009.

[6] T. Yagi et al., "Background self-calibration algorithm for pipelined ADC using split ADC scheme," IEICE Trans. Electron., vol. E94-C, no. 7, pp. 1233-1236, 2011.

[7] B. Razavi, "Design considerations for interleaved ADCs," IEEE Journal of Solid State Circuits, vol. 48, no. 8, pp. 1806-1817, 2013.

[8] B. D. Sahoo and B. Razavi, "A 12-Bit 200-MHz CMOS ADC," IEEE Journal of Solid State Circuits, vol. 44, no. 9, pp. 2366-2380, 2009.

[9] S. K. Shin et al., "A 12 bit 200MS/s zero-crossing-based pipelined ADC with early sub-ADC decision and output residue background calibration," IEEE Journal of Solid State Circuits, vol. 49, no. 6, pp. 1366-1381, 2014.

[10] K. Honda, M. Furuta and S. Kawahito, "A low-power low-voltage 10-bit 100-MSamples/s pipeline A/D Converter Using Capacitance Coupling Techniques." IEEE Journal of Solid State Circuits, vol. 42, no. 4 , pp. 757-765, 2007

[11] A. A. Ali et al., "A 16-bit 250-MS/s IF sampling pipelined ADC with background calibration," IEEE Journal of Solid State Circuits, vol. 45, no. 12, pp. 2602-2612, 2010.

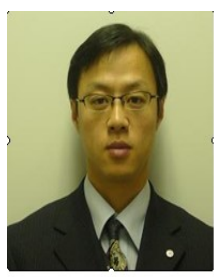

Haijun Lin was born in Wulumuqi, China. He received B.S, M.S and Ph.D. degrees in electronic engineering from Gunma University, Japan in 2004, 2006 and 2010, respectively.

He worked for Freescale Semiconductor Japan in 2006 as a design engineer. He joined Fujitsu Laboratories Limited as a researcher in Japan in 2010. Since September 2011, he worked as an associate professor in the Department of Electrical and Electronics Engineering in Xiamen University of Technology. His current research interests include high frequency analog filters, high speed and high resolution $\mathrm{AD}$ converters, analog front-end of very high speed data receiver and all digital PLLs.

$\mathrm{He}$ is the reviewer of the Institute of Electronics, Information and Communication Engineers (IEICE).

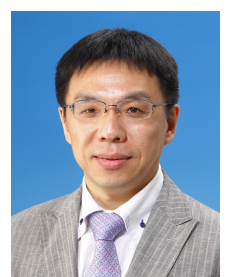

Hao San was born in Liaoning, China. He received B.S degree from Liaoning Institute of Technology, China in 1993, M.S and Ph.D. degrees in electronic engineering from Gunma University, Japan in 2000 2004 respectively.

From 2000 to 2001, he worked with Kawasaki Microelectronics, Inc. He joined Gunma University as an assistant professor in the Department of Electronic Engineering in 2004. In 2009, he joined Tokyo City University as an associate professor in the Department of Information Network Engineering. His research interests include analog and mixed-signal integrated circuits.

Dr. San has been an Associate Editor of IEICE Transactions on Electronics from 2010. He was the treasurer of the IEEE Circuits and Systems Society Japan Chapter from 2011 to 2012. He is the member of IEEE and IEEJ.

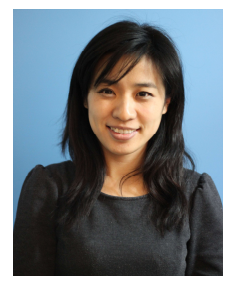

Ye Tian was born in Jinan, China. She received B.S degree in electronic engineering from Shandong University of Industry, China, in 1999.

From 1999 to 2005, she worked as a testing engineer with Beijing Tiancheng Electronics Technology Co., Ltd, Beijing, China. She joined Xiamen Hualian Electronics Co. Ltd as a system engineer in 2005, Xiamen, China. In 2013, she joined Xiamen Xunxin Electronics Technology Co. Ltd. Xiamen, China as the testing engineer.

Miss Tian's research interests include high speed and high resolution $\mathrm{ADC}$ testing, system testing of high speed receiver. 